\title{
Linfangiomatosis Kaposiforme: Reporte de un Caso y Revisión de la Literatura
}

\author{
Kaposiform Lymphangiomatosis: A Case Report and Literature Review
}

Enmanuel Guerrero ${ }^{1,2,3}$; Raúl Alvarado ${ }^{1}$ Pablo Monsalve ${ }^{1}$; Marx Bravo ${ }^{1}$; Adriana Urdiales ${ }^{1}$; Carlos Manterola ${ }^{3,5}$ \& Lorena Albarracín ${ }^{4}$

\begin{abstract}
GUERRERO, E.; ALVARADO, R.; MONSALVE, P.; BRAVO, M.; URDIALES, A.; MANTEROLA, C. \& ALBARRACÍN, L. Linfangiomatosis kaposiforme: reporte de un caso y revisión de la literatura. Int. J. Morphol., 38(4):1060-1064, 2020.

RESUMEN: La linfangiomatosis es una patología benigna, de etiología desconocida, que afecta especialmente a población pediátrica, caracterizándose por desarrollo de masas subcutáneas que involucran uno o más órganos. Cuando se asocia con coagulopatía y derrames quilosos, recibe el nombre de linfangiomatosis kaposiforme (LK), cuya evidencia disponible es escasa. El objetivo de este manuscrito es reportar un caso de LK pediátrico atendido en el Instituto del Cáncer SOLCA, Cuenca, Ecuador. Paciente femenina de 1 año 7 meses, con hernia inguinal bilaterales y alteración de los tiempos de coagulación. En los estudios de imagen se evidenció una masa mediastínica asociada a presencia de líquido en espacio pleural y cavidad abdomino-pélvica. Además, se evidenció la existencia de múltiples imágenes hipogénicas en el bazo. Se practicó timectomía mínima, toma de biopsia de linfonodos mediastínicos para establecer el diagnóstico, y se instaló un dren en el tórax para tratar el derrame pleural antes señalado. Se descartaron patologías similares (linfomas, lupus, inmunológicas, etc.). Dado el continúo deterioro respiratorio, coagulopatía, lesiones hipogénicas esplénicas y drenaje abundante del quilotórax por el tubo torácico, se planteó el diagnóstico de LK. Se inició apoyo nutricional vía parenteral (sin lípidos); se indicó vincristina, y se realizó una pleurodesis con bleomicina sin buena respuesta. La paciente falleció a los 16 días de su ingreso. Se trata de un caso de mal pronóstico, con evolución rápidamente progresiva hacia un desenlace fatal.
\end{abstract}

PALABRAS CLAVE: Linfangiomatosis; Coagulopatía; Derrame pleural; Linfangiomatosis kaposiforme.

\section{INTRODUCCIÓN}

La linfangiomatosis es una malformación congénita de curso clínico benigno, poco frecuente y de etiología desconocida. Se presenta generalmente durante la infancia y se diagnostica en las primeras dos décadas de la vida (Ji et al., 2019).

En ocasiones, se puede complicar por proliferación linfática multifocal (mediastino, pulmones, pleura, sistema óseo y torácico); y asociación a derrames pleurales y pericárdicos (Ji et al.).

Cuando se acompaña de coagulopatía y derrames quilosos, se denomina linfangiomatosis kaposiforme (LK); entidad nosológica de mal pronóstico, de elevada morbimortalidad, cuya complicación más frecuente es el deterioro de la función respiratoria (Raam et al., 2016; Adams et al., 2018).
El objetivo de este manuscrito fue reportar un caso de LK pediátrico atendido en Instituto del Cáncer SOLCA, Cuenca, Ecuador.

Este artículo fue escrito siguiendo la iniciativa CARE (case report guidelines) para el reporte de casos clínicos (Gagnier et al., 2014).

\section{CASO CLÍNICO}

Se trata de una paciente de 1 año 7 meses, de $12 \mathrm{~kg}$ de peso, sin antecedentes personales ni familiares relevantes. Cinco meses antes de su ingreso, se le diagnosticó hernia inguinal bilateral, razón por la cual se le realizaron estudios pre-quirúrgicos en los que se encontró alteración del

\footnotetext{
${ }^{1}$ Instituto del Cáncer SOLCA, Cuenca, Ecuador.

${ }^{2}$ Facultad de Medicina, Universidad de Cuenca, Ecuador.

${ }^{3}$ Programa de Doctorado en Ciencias Médicas, Universidad de La Frontera, Temuco, Chile.

${ }^{4}$ Servicios Médicos Integrales

${ }^{5}$ Centro de Estudios Morfológicos y Quirúrgicos (CEMyQ), Universidad de La Frontera, Temuco, Chile.
} 
tiempo de coagulación; motivo por la que fue derivada al Instituto SOLCA, Cuenca.

Al ingresar a esta Institución, se realizó un ultrasonido abdominal (US), en el que pudo verificarse un bazo con imágenes hipoecoicas de 4,8 y 6,2 mm; y la presencia de líquido libre en el espacio pleural derecho y la cavidad abdominal. Además, se constató la existencia de imágenes quísticas a nivel subdiafragmático derecha de 22 por $12 \mathrm{~mm}$ (Fig. 1).

Se realizaron además, pruebas diagnósticas generales, entre las que destacaron hipoproteinemia, hipofibrinógenemia, alteración de la función hepática, neutropenia y dímero D elevado.

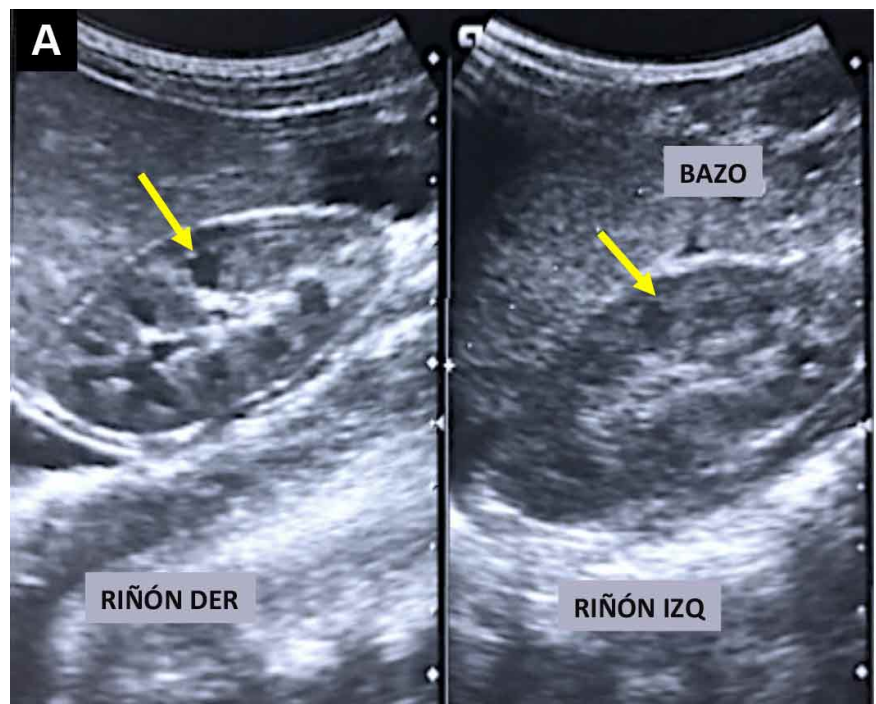

El aspirado de médula ósea y los estudios de citometría de flujo descartaron infiltración por enfermedades linfoproliferativas.

La tomografía axial computarizada (TAC) de tórax y abdomen, permitió evidenciar la existencia de una imagen nodular e hipodensa, de 37 por $23 \mathrm{~mm}$ de diámetro, localizada en el mediastino; y líquido libre en el espacio pleural derecho. A nivel abdominal, se verificó la presencia de múltiples imágenes hipodensas redondeadas, de distribución difusa, de entre 3 y 10 mm de diámetro, localizadas en el bazo; y abundante líquido libre en la cavidad abdominal y pélvica (Fig. 2).

Con el objeto de establecer diagnóstico, se realizó biopsia de la masa mediastínica (timectomía mínima más toma de

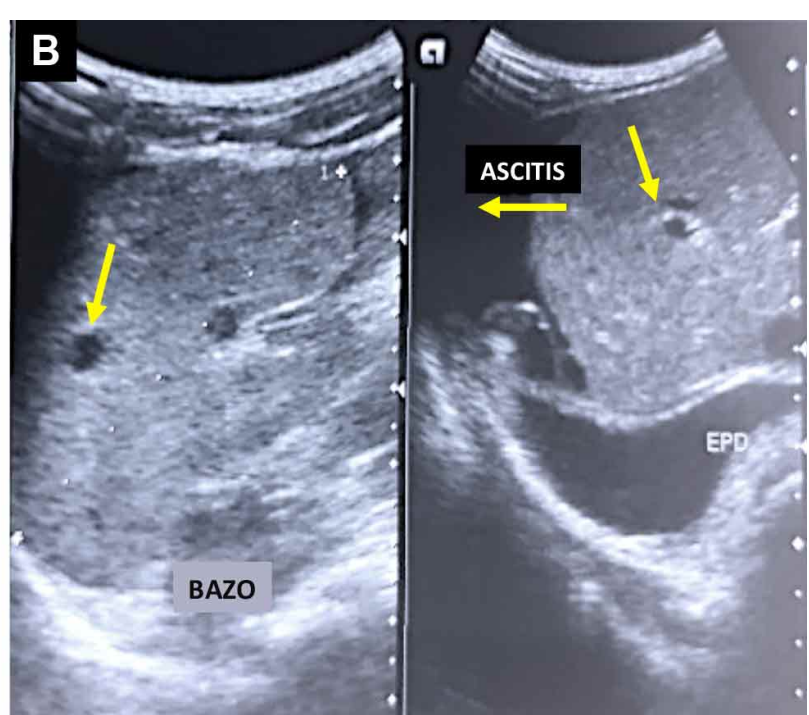

Fig. 1. Ecotomografía abdominal de la paciente. Se puede apreciar el bazo con múltiples imágenes hipoecoicas (1A). Además, la existencia de moderada cantidad de líquido libre en el espacio pleural derecho y en la cavidad abdominal, con imágenes quísticas en región subdiafragmática derecha (1B).
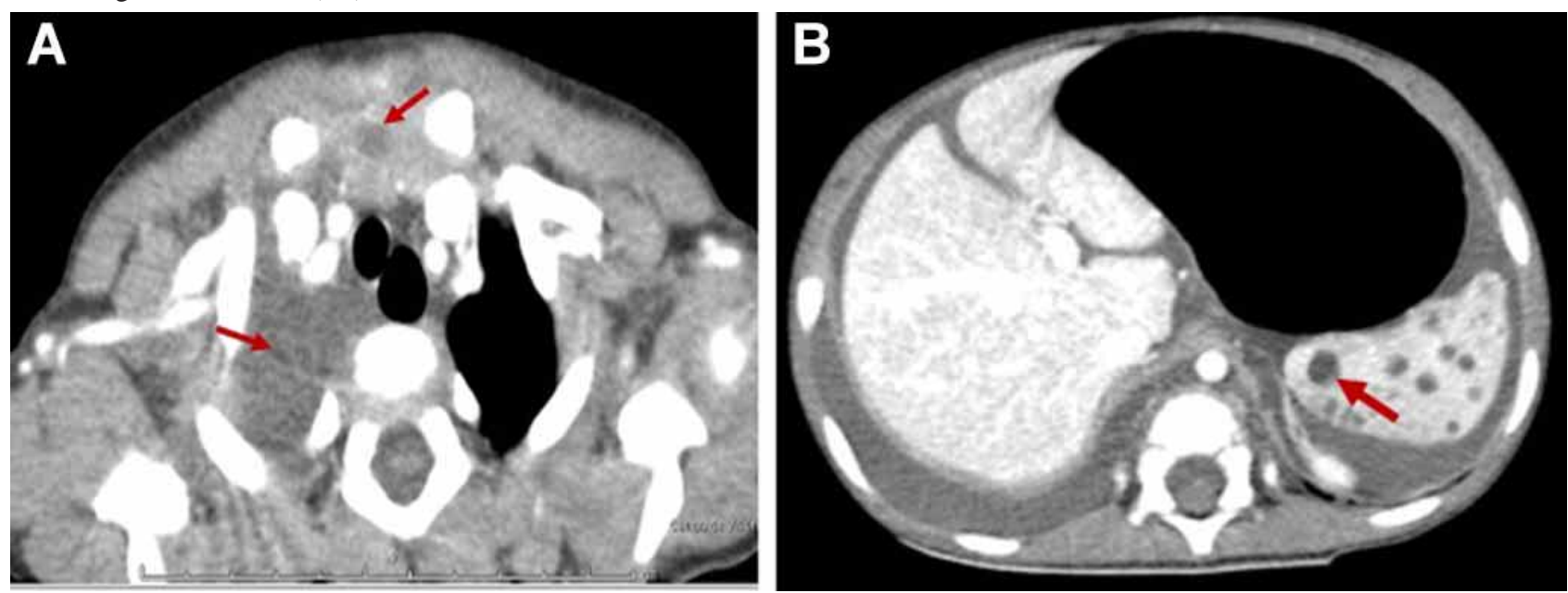

Fig. 2. Tomografía de tórax y abdomen, en la que se puede verificar una imagen mediastínica, de aspecto nodular, hipodensa, sin realce al contraste y derrame pleural derecho no loculado (2A). Al margen derecho, se observa el bazo, con múltiples imágenes hipodensas, redondeadas, y ascitis. 
muestras para estudio histológico de linfonodos mediastínicos); cuyo resultado fue de histología normal (Fig. 3).

La paciente cursó con insuficiencia respiratoria secundaria a un síndrome de ocupación pleural derecha, de carácter masivo; razón por la cual fue necesario realizar una toracostomía con tubo, medida que permitió el drenaje de 250 cc de líquido seroso, compatible con quilotórax, con un débito promedio de 350cc/día durante 16 días (Fig. 4).
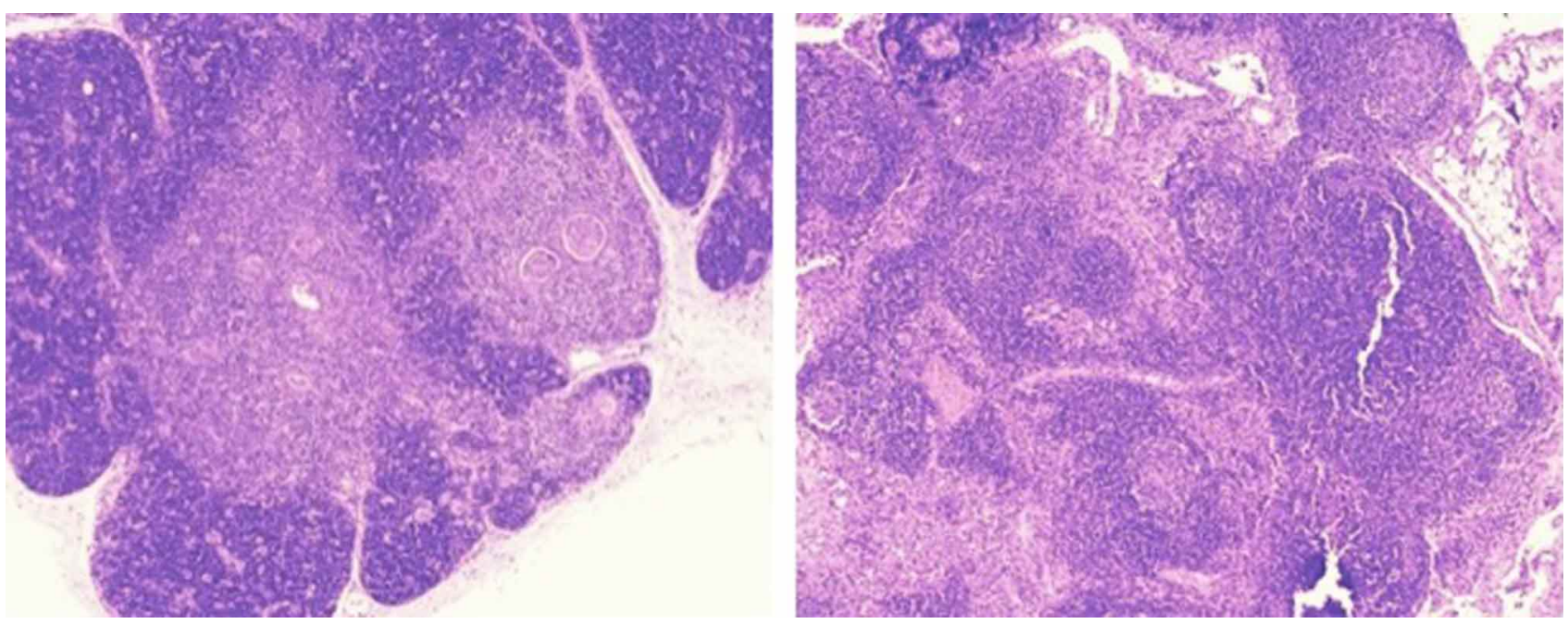

Fig. 3. Cortes histológicos con tinción con hematoxilina eosina; en los que se visualiza el parénquima tímico rodeado de una cápsula de tejido conectivo laxo. Dividido en lóbulos y lobulillos, por tabiques fibrosos y organizados en dos compartimientos: corteza y médula.

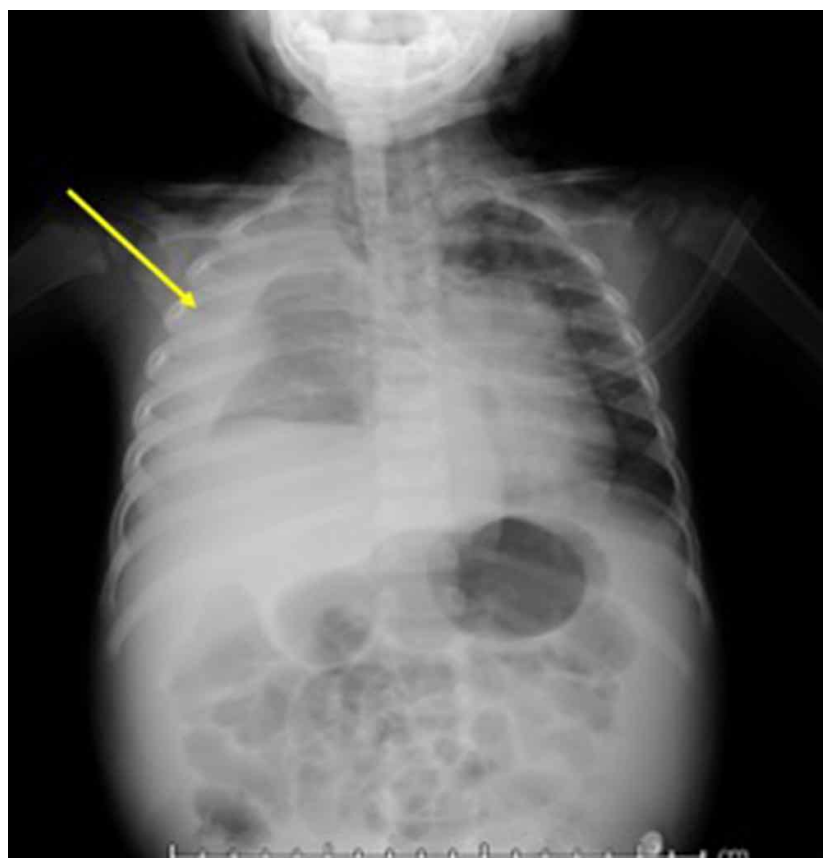

Fig. 4. Radiología simple toraco-abdominal, en la que se puede constatar la existencia de un hidroneumotórax masivo en el hemitórax derecho.

Se descartaron procesos oncológico, inmunológico y reumatológico, mediante la realización de múltiples pruebas diagnósticas serológicas, de imágenes, y estudios patológicos complementarios.
La paciente siguió deteriorándose desde el punto de vista general, los síntomas respiratorios se fueron agravando progresivamente. El quilotórax continuó siendo masivo. Lo que, asociado a la existencia de las lesiones esplénicas y subdiafragmáticas antes descritas; permitieron plantear el diagnóstico de LK.

En virtud de ello, se decidió instaurar tratamiento específico para LK, que consistió en nutrición parenteral parcial sin lípidos; pleurodesis con bleomicina $\left(15 \mathrm{U} / \mathrm{m}^{2}\right)$ dos veces por semana; administración de vincristina $(1,5$ $\left.\mathrm{mg} / \mathrm{m}^{2}\right)$ vía parenteral; y propanolol $(0,8 \mathrm{mg} / \mathrm{kg} /$ día $)$ via oral. Además, fue necesario administrar otras medidas terapéuticas de soporte: transfusión de plasma y crioprecipitados; antibióticos (meropenen+vancomicina), antifúngicos, bolos de corticoesteroides, etc. Sin objetivarse respuesta clínica favorable.

Lamentablemente, la evolución fue desfavorable. La paciente desarrolló una coagulación intravascular diseminada, síndrome de distrés respiratorio, arritmia, sepsis urinaria por cándida tropicalis, shock séptico refractario, y disfunción multiorgánica. Luego de 16 días la paciente falleció.

La necropsia confirmó el diagnóstico de LK con compromiso de ambos pulmones, corazón y bazo. 


\section{DISCUSIÓN}

La etiología de la linfangiomatosis es desconocida, pero parece deberse a una anormalidad del desarrollo linfático y la presencia de múltiples linfangiomas (tejido linfático bien diferenciado que se presenta como acumulaciones quísticas) (Ozeki \& Fukao, 2019; Ozeki et al., 2019a).

Se clasifica en diferentes fenotipos como: anomalía linfática generalizada, enfermedad de Gorham Stout y LK (Luisi et al., 2016; Ozeki \& Fukao).

Las manifestaciones clínicas son inespecíficas y puede involucrar a un solo órgano (por ejemplo: linfangiomatosis pulmonar difusa), o a múltiples órganos. A nivel toracico, puede afectar pulmones, mediastino, corazón, pleura, conducto y pared torácica (Hu \& Zhou, 2018).

Se caracteriza histológicamente por células endoteliales linfáticas en huso y una variante oncogénica NRAS en algunas muestras de ADN (Barclay et al., 2019; Ozeki et al., 2019b). Esta paciente presentó un diagnóstico clínico de LK, debido a la rápida progresión de disnea, derrames quilosos y anomalías de la coagulación.

La LK incluye síntomas respiratorios (50-100 \%), coagulopatía (50-100 \%) y masa mediastína $(82 \%)$. Los problemas respiratorios más frecuentes son tos, disnea y derrames pleurales (Croteau et al., 2014; Ozeki et al., 2019a). La coagulopatía puede ser causada por trombocitopenia, producción local de fibrinolisina o malformaciones venosas (Luisi et al.).

El deterioro multiorgánico y los hallazgos antes descritos en las pruebas de imagen (US y TAC) son suficientes para plantear el diagnóstico de LK, por lo que la toma de muestras para estudio histológico podría evitarse (Raam et al.; Iacobas et al., 2020).

La linfangiografía, es útil para estudiar la estructura del sistema linfático, por su precisión en la identificación de la extensión de las lesiones (Iacobas et al.). En este caso, el deterioro progresico y grave del estado de salud de la paciente, no permitió su realización.

El tratamiento es paliativo y tiene como objetivo solamente retrasar la progresión de la enfermedad y aliviar los síntomas relacionados a la compresión de las estructuras adyacentes y a la acumulación de líquido quiloso.

No existen protocolos o pautas de tratamiento estandarizados (Luisi et al.; Adams et al.), posiblemente debido a lo infrecuente de la enfermedad, y por ende a la escasa evidencia existente respecto de ella.

Los pocos estudios existentes recomiendan medidas dietéticas con nutrición parenteral sin lípidos o con triglicéridos de cadena media, así como dietas altas en proteínas, aunque en general han demostrado poca efectividad.

Cuando la LK se localizada a nivel torácico, se puede considerar la indicación de un acceso quirúrgico (idealmente por videotoracoscopía), especialmente si existen síntomas de ocupación pleural (Luisi et al.; Ozeki et al., 2019a). Para reducir el derrame pleural recurrente, esta indicado la pleurectomía parietal, ligadura del conducto torácico o la pleurodesis con 15 a $120 \mathrm{U}$ de bleomicina intracavitario diluida en $100 \mathrm{cc}$ de solusión salina y removida 6 a 24 horas después (Bagheri et al., 2018).

El uso de propanolol (como angiogénico y linfangiogénico), a dosis crecientes ( 1 a $4 \mathrm{mg} / \mathrm{kg} / \mathrm{día}$ ), puede reducir el derrame pleural. Sin embargo, el escaso conocimiento existente de la etiología y patogénesis de esta enfermedad impide lograr avances terapéuticos reales (Luisi et al.; Adams et al.; Ji et al., 2018).

La radioterapia, quimioterapia sistémica (vincristina $1,5 \mathrm{mg} / \mathrm{m}^{2}$, ciclofosfamida $\left.300 \mathrm{mg} / \mathrm{m}^{2}\right)$, los esteroides, el bevacizumab ( $1 \mathrm{mg} / \mathrm{kg}$ cada 3 semanas) y el interferón sistémico, se han utilizado con resultados poco satisfactorios (Croteau et al.; Luisi et al.). La única medida terapéutica que cuenta con alguna evidencia que la apoya, es la administración del inhibidor de mTOR sirolimus; que ha demostrado eficacia en el control de la enfermedad, buena tolerancia y escasos efectos secundarios (Ozeki \& Fukao; Ricci et al., 2019; Ozeki et al., 2019c).

Lo que está meridianamente claro, es que ante la sospecha clínica de LK es importante un tratamiento multidisciplinario debido a sus múltiples y graves complicaciones (Adams et al.; Ji et al., 2019).

El pronóstico de esta patología es desfavorable y depende de la extensión de la enfermedad. Cuando causa osteólisis masiva, se asocia a una alta morbilidad y mortalidad por infección, parálisis, empeoramiento de la función pulmonar y derrames quilosos (Luisi et al.; Ozeki et al., 2019a). En esta infante, la enfermedad afectaba varios órganos vitales lo que le ocasionó su deceso.

Dada la baja prevalencia e incidencia de la LK, no existen datos epidemiólogicos. Es por ello, que nos parece relevante aportar información con este caso, pues incrementa el escaso conocimiento existente. 
A modo de conclusión, se puede señalar que se trata de un caso de mal pronóstico, con evolución rápidamente progresiva hacia un desenlace fatal.

Responsabilidades éticas. Protección de personas y animales: Los autores declaran que los procedimientos seguidos se conformaron a las normas éticas del comité de experimentación humana responsable y de acuerdo con la Asociación Médica Mundial y la Declaración de Helsinki.

Confidencialidad de los datos: Los autores declaran que han seguido los protocolos de su centro de trabajo sobre la publicación de datos de pacientes.

Derecho a la privacidad y consentimiento informado: Los autores han obtenido el consentimiento informado de los padres de la paciente referida en este artículo. Este documento obra en poder del primer autor.

AGRADECIMIENTOS. Agradecemos al personal del Instituto del Cáncer SOLCA, Cuenca, Ecuador.

GUERRERO, E.; ALVARADO, R.; MONSALVE, P.; BRAVO, M.; URDIALES, A.; MANTEROLA, C. \& ALBARRACÍN, L. Kaposiform lymphangiomatosis: a case report and literature review. Int. J. Morphol., 38(4):1060-1064, 2020.

SUMMARY: Lymphangiomatosis is a benign pathology, of unknown etiology. Affects especially to pediatric population and is characterized by development of subcutaneous masses that involve one or more organs. When it presents with coagulopathy and chylous effusions, it is called kaposiform lymphangiomatosis (KL). The available evidence of KL is scarce. The objective of this manuscript was to report a case of pediatric KL treated at SOLCA Cancer Institute, Cuenca, Ecuador. Female patient of 1 year and 7 months age. She had a bilateral inguinal hernia and altered coagulation times. Imaging studies revealed a mediastinal mass associated with fluid in the pleural space and in the abdominalpelvic cavity. Furthermore, the existence of multiple hypogenic images in the spleen was evidenced. Minimal thymectomy, biopsy of mediastinal lymph nodes was performed to establish the diagnosis, and a drain was installed in the chest to treat the aforementioned pleural effusion. Other pathologies like lymphomas, lupus, etc. were ruled out. Given the continuous respiratory deterioration, coagulopathy, splenic hypogenic lesions and abundant drainage of the chylothorax through the chest tube, the diagnosis of KL was raised. Parenteral nutrition (lipid-free) was started; Vincristine was indicated, and a pleurodesis with bleomycin was performed without a good response. The patient died 16 days after her admission. It is a case of very bad prognosis, with a rapidly progressive evolution towards a fatal outcome.

KEY WORDS: Lymphangiomatosis; Coagulopathy; Pleural effusion; Kaposiform lymphangiomatosis.

\section{REFERENCIAS BIBLIOGRÁFICAS}

Adams, D. M.; Brandão, L. R.; Peterman, C. M.; Gupta, A.; Patel, M.; Fishman, S. $\&$ Trenor, C. C. Vascular anomaly cases for the pediatric hematologist oncologists-An interdisciplinary review. Pediatr. Blood Cancer, 65(1):e26716, 2018.

Bagheri, R.; Noori, M.; Rajayi, M.; Attaran, D.; Mohammad Hashem Asna Ashari, A.; Mohammadzadeh Lari, S.; Basiri, R.; Rezaeetalab, F.; Afghani, R. \& Salehi, M. The effect of iodopovidone versus bleomycin in chemical pleurodesis. Asian Cardiovasc. Thorac. Ann., 26(5):382-6, 2018.

Barclay, S. F.; Inman, K. W.; Luks, V. L.; McIntyre, J. B.; Al-Ibraheemi, A.; Church, A. J.; Perez-Atayde, A. R.; Mangray, S.; Jeng, M.; Kreimer, S. R.; et al. A somatic activating NRAS variant associated with kaposiform lymphangiomatosis. Genet. Med., 21(7):1517-24, 2019.

Croteau, S. E.; Kozakewich, H. P.; Perez-Atayde, A. R.; Fishman, S. J.; Alomari, A. I.; Chaudry, G.; Mulliken, J. B. \& Trenor, C. C. 3rd. Kaposiform lymphangiomatosis: a distinct aggressive lymphatic anomaly. J. Pediatr., 164(2):383-8, 2014

Gagnier, J. J.; Kienle, G.; Altman, D. G.; Moher, D.; Sox, H.; Riley, D. \& CARE Group. The CARE guidelines: consensus-based clinical case report guideline development. J. Clin. Epidemiol., 67(1):46-51, 2014.

Hu, P. A. \& Zhou, Z. R. Clinical and imaging features of Kaposiform Hemangioendothelioma. Br. J. Radiol., 91(1086):20170798, 2018.

Iacobas, I.; Adams, D. M.; Pimpalwar, S.; Phung, T.; Blei, F.; Burrows, P.; LopezGutierrez, J. C.; Levine, M. A. \& Trenor, C. C. 3rd. Multidisciplinary guidelines for initial evaluation of complicated lymphatic anomalies-expert opinion consensus. Pediatr. Blood Cancer, 67(1):e28036, 2020.

Ji, Y.; Chen, S.; Li, L.; Yang, K.; Xia, C.; Li, L.; Yang, G.; Kong, F.; Lu, G. \& Liu, $\mathrm{X}$. Kaposiform hemangioendothelioma without cutaneous involvement. $J$. Cancer Res. Clin. Oncol., 144(12):2475-84, 2018.

Ji, Y.; Chen, S.; Peng, S.; Xia, C. \& Li, L. Kaposiform lymphangiomatosis and kaposiform hemangioendothelioma: similarities and differences. Orphanet $J$. Rare Dis., 14(1):165, 2019.

Luisi, F.; Torre, O. \& Harari, S. Thoracic involvement in generalised lymphatic anomaly (or lymphangiomatosis). Eur. Respir. Rev., 25(140):170-7, 2016.

Ozeki, M. \& Fukao, T. Generalized lymphatic anomaly and gorham-stout disease: overview and recent insights. Adv. Wound Care (New Rochelle), 8(6):230-45, 2019.

Ozeki, M.; Aoki, Y.; Nozawa, A.; Yasue, S.; Endo, S.; Hori, Y.; Matsuoka, K.; Niihori, T.; Funayama, R.; Shirota, M.; et al. Detection of NRAS mutation in cell-free DNA biological fluids from patients with kaposiform lymphangiomatosis. Orphanet J. Rare Dis., 14(1):215, $2019 \mathrm{~b}$.

Ozeki, M.; Nozawa, A.; Kawamoto, N.; Fujino, A.; Hirakawa, S. \& Fukao, T. Potential biomarkers of kaposiform lymphangiomatosis. Pediatr. Blood Cancer, 66(9):e27878, 2019a.

Ozeki, M.; Nozawa, A.; Yasue, S.; Endo, S.; Asada, R.; Hashimoto, H. \& Fukao, T The impact of sirolimus therapy on lesion size, clinical symptoms, and quality of life of patients with lymphatic anomalies. Orphanet J. Rare Dis., 14(1):141, 2019c.

Raam, M. S.; Festekjian, A. \& Elkhunovich, M. A. Point-of-care thoracic ultrasonography in the diagnosis and management of kaposiform lymphangiomatosis. Pediatr. Emerg. Care, 32(12):888-91, 2016.

Ricci, K. W.; Hammill, A. M.; Mobberley-Schuman, P.; Nelson, S. C.; Blatt, J.; Bender, J. L. G.; McCuaig, C. C.; Synakiewicz, A.; Frieden, I. J. \& Adams, D. M. Efficacy of systemic sirolimus in the treatment of generalized lymphatic anomaly and Gorham-Stout disease. Pediatr. Blood Cancer, 66(5):e27614, 2019.

Dirección para correspondencia:

Dr. Carlos Manterola

CEMyQ

Universidad de La Frontera

Temuco - CHILE

Recibido: $10-01-2020$

Aceptado: 15-02-2020

Email: carlos.manterola@ufrontera.cl 